\title{
Difference in TRI13 Gene Sequences between the 3-Acetyldeoxynivalenol Producing Fusarium graminearum Chemotypes from Canada and China
}

\author{
Chami Amarasinghe ${ }^{1}$, Jian-Hua Wang ${ }^{2}$, Yu-Cai Liao ${ }^{2,3}$ and W.G. Dilantha Fernando ${ }^{1, *}$ \\ 1 Department of Plant Science, University of Manitoba, Winnipeg, MB R3T2N2, Canada; \\ E-Mail: umamarac@cc.umanitoba.ca \\ 2 Molecular Biotechnology Laboratory of Triticeae Crops, Huazhong Agricultural University, \\ Wuhan 430070, Hubei, China; E-Mails: jianhuawang163@163.com (J.H.W.); \\ yucailiao@mail.hzau.edu.cn (Y.-C.L.) \\ 3 College of Plant Science and Technology, Huazhong Agricultural University, Wuhan 430070, \\ Hubei, China
}

* Author to whom correspondence should be addressed; E-Mail: d_fernando@umanitoba.ca; Tel.: +1-204-4746072; Fax: +1-204-474-7528.

Received: 25 August 2011; in revised form: 9 September 2011 / Accepted: 9 September 2011 / Published: 20 September 2011

\begin{abstract}
Positive-negative PCR assays based on the genes involved in the trichothecene biosynthesis pathway are useful in assessing the risk of trichothecene contamination in grain and are important in epidemiological studies. A single PCR detection method based on the structural gene sequence of TRII3 gene has been developed to predict the 3-ADON, 15-ADON and NIV chemotypes in China. The chemotypic differences are based on the deletions within the TRI13 gene. The objective of this study was to assess the reliability of using this single primer based on the TRI13 gene to differentiate the F. graminearum chemotypes in Canada. In this study, we found that, this single PCR detection method based on the deletions in the TRI13 gene cannot be used to differentiate the 3-ADON and 15-ADON chemotypes in the Canadian F. graminearum isolates; further sequence analysis of the PCR products confirmed that both Canadian 3-ADON and $15-\mathrm{ADON}$ chemotypes have the $61 \mathrm{bp}$ deletion in the TRI13 gene. This $61 \mathrm{bp}$ deletion was absent in the Chinese 3-ADON isolates. Therefore these findings revealed that there are genetic differences between the examined 3-ADON F. graminearum isolates from Canada and China. The observed genetic differences between the 3-ADON chemotype populations
\end{abstract}


in Canada and China may be resulted from a random mutation (insertion/deletion) that took place in one of the populations and accumulated due to genetic drift and/or selection.

Keywords: Fusarium graminearum; chemotypes; PCR

\section{Introduction}

Fusarium head blight (FHB) is an economically important disease worldwide. Apart from causing significant yield losses the fungus can produce trichothecene mycotoxins. Harvested grains, contaminated with trichothecene mycotoxins can cause both acute and chronic side effects in livestock and humans. It has been reported that $25 \%$ of the world crop is affected by mycotoxins [1]. In plants, these mycotoxins have been shown to act as virulence factors during pathogenesis [2]. There are mainly two classes of trichothecenes; class A and class B, class B contains a keto group at the C-8 position of the trichothecene ring [3]. Different Fusarium spp such as, F. graminearum, F. culmorum and $F$. cerealis can produce different types of class B trichothecenes, such as deoxynivalenol (DON), nivalenol (NIV) and their acetylated derivatives, 3-acetyldeoxynivalenol (3-ADON), 15-acetyldeoxynivalenol (15-ADON) and 4-acetylnivalenol (4-ANIV). Based on type B tricothecene production, three different chemotypes of $F$. graminearum have been identified. The 3-ADON chemotype produces both DON and 3-ADON; 15-ADON chemotype produces both DON and 15-ADON; and the NIV chemotype produces NIV and 4-ANIV [3]. The differences between chemotypes are governed by the different genes involved in the trichothecene biosynthesis pathway. Trichothecene biosynthesis is a complex process, mediated by at least 10 genes that are involved in a series of oxygenation, isomerization and esterification steps [4]. The trichothecene gene cluster includes trichodiene synthase (TRI5), P450 oxygenase (TRI4 and TRI11), acetyltransferases (TRI3 and TRI7), transcription factors (TRI6 and TRI10), a toxin efflux pump (TRI12), and two unidentified hypothetical proteins (TRI8 and TRI9). The acetyltransferase gene TRI101, unlinked to the TRI cluster, is also found to be involved in the biosynthesis pathway. Two genes TRI13 and TRII4 have been identified from $F$. sporotrichiodies [4,5]. Among the TRI cluster genes TRII3 is found to be the determinant for the DON-NIV switching in Fusarium and TRI7 is involved in further modification of NIV to its acetylated derivative 4-ANIV [5]. NIV chemotypes have functional copies of TRI13 and TRI7 genes whereas both genes are non-functional in DON chemotypes [5].

Various PCR based assays have been developed to examine the trichothecene mycotoxin genotypes of $F$. graminearum. These assays are based on the amplification of a part of a gene that encodes for key enzymes involved in the trichiothecene biosynthesis pathway [6-9]. These PCR based assays provide a rapid and cost-effective way of identifying the different $F$. graminearum chemotypes [7].

The genotypic differences among the $F$. graminearum isolates collected from different populations in diverse geographic regions have been extensively studied [10]. To date, 13 phylogenetically distinct species have been identified within the $F$. graminearum clade based on DNA sequences from 13 genes [11-14]. These lineages are morphologically cryptic. F. graminearum sensu stricto (lineage 7) is the predominant species found in North America [15]. In China, F. asiaticum (lineage 6) and F. graminearum sensu stricto (lineage 7) are the most prevalent FHB causing species [16]. 
O'Donnell et al. [10] and Ward et al. [7] reported that the evolution of genes involved in the B-tricothecene biosynthesis does not correlate with the F. graminearum clade phylogeny. It is believed that chemotype polymorphism is trans-specific and has been maintained through multiple speciation events by balancing selection [7]. High levels of genetic variations have been found in the virulence associated genes within the $F$. graminearum clade [7]. The different lineages of $F$. graminearum have different geographic distributions; also differ in the type of mycotoxins that they produced and they may differ in their ability to cause disease on particular crops [17]. Environment factors within a certain geographical area could influence the prevalence of a particular chemotype. Zhang et al. [16] investigated the mycotoxin chemotype frequency of the $F$. graminearum strains isolated from wheat in FHB epidemic regions of China. The investigated chemotypes appeared to have different geographical distribution patterns and these were associated with the annual average temperatures in the region [17]. $\mathrm{Qu}$ et al. [18] also investigated the geographical distribution of $F$. graminearum and $F$. asiaticum isolates from all regions in China and demonstrated that $F$. graminearum isolates were more prevalent in the cooler regions whereas $F$. asiaticum in the warmer regions.

Ward et al. [7] reported the impact of environment on pathogen fitness. It has been reported that the chemotypes of $F$. graminearum from China and North America were different [3,19]. Miller et al. [3], analyzed the metabolic profiles of $F$. graminearum strains using gas chromatography/mass spectroscopy and reported that, chemotype $1 \mathrm{~A}(\mathrm{DON}$ and 3-ADON) was common in China and chemotype 1B (DON and 15-ADON) in North America. The results from phylogenetic studies are important to prevent the unintentional introduction of species or genetically different strains from one genetically unique population to another population especially with international trade [20]. The introduction of new Fusarium species to the existing population may affect the current management strategies and breeding programs.

Therefore studies on the genetic diversity of $F$. graminearum chemotypes found in different geographical regions are important to prevent the future FHB epidemics. Wang et al. [21] developed a generic PCR detection method based on the TRI13 gene to identify 3-ADON, 15-ADON and NIV chemotypes using a single primer pair. The objective of this study is to examine the applicability and reliability of this generic PCR detection based on TRI13 gene to identify the chemotypes of F. graminearum isolates from Canada.

\section{Materials and Methods}

\subsection{Fungal Isolation and DNA Extraction}

F. graminearum isolates collected from different regions of Canada (120 isolates) and China (39 isolates) were used (Table 1). Some of the Canadian isolates were identified using multiplex PCR by Guo et al. [22] and the others by Dr.'s Ward and O'Donnell at the United States Department of Agriculture, Peoria, IL, using a multilocus genotyping assay. Chinese isolates were identified in Dr.'s $\mathrm{Xu}$ Zhang and Hongxiang Ma's labs, at the Jiangsu Academy of Agriculture Science, China.

Single spore cultures of $F$. graminearum isolates growing from the fusarium damaged kernels were used for the DNA extraction. All isolates were grown on potato dextrose agar (PDA) plates for 7 days and genomic DNA was extracted from the freeze dried aerial mycelium using a CTAB based protocol 
described by Fernando et al. [23]. DNA was quantified using the NanoDrop3300 (ThermoFisher Scientific Inc.). DNA was diluted using sterilized distilled water for final concentration of $50 \mathrm{ng} / \mu \mathrm{L}$.

Table 1. Description of location, host and number of Fusarium graminearum isolates used in this study.

\begin{tabular}{|lcc|}
\hline Location & Host & Number of Isolates \\
\hline Manitoba, Canada & Wheat & 93 \\
Saskatchewan, Canada & Wheat & 15 \\
Nova Scotia, Canada & Wheat & 3 \\
Alberta, Canada & Wheat & 4 \\
Prince Edward Island, Canada & Wheat & 2 \\
Quebec, Canada & Wheat & 3 \\
China & Wheat & 39 \\
\hline
\end{tabular}

\subsection{SCAR Analysis}

All the Fusarium strains were subjected to SCAR (sequence characterized amplified region) analysis with a pair of primers Fg16F/R [16,24]. These primers generate a 410 bp DNA fragment specific for SCAR group I and a 497 bp fragment for SCAR group V, respectively [16].

\subsection{PCR Assays for Trichothecene Chemotypes}

Two PCR assays were carried out. In the first PCR, the fungal DNA was amplified using the single primer set, developed by Wang et al. [21]. The single primer set used for amplification was as follows: TRI13P1 (5'-CTCSACCGCATCGAAGASTCTC-3') and TRI13P2 (5'-GAASGTCGCARGACCTTGTTTC-3'), these primers generate a 859 bp fragment from NIV producing strains, a $644 \mathrm{bp}$ fragment from $3-\mathrm{ADON}$ producers and a $583 \mathrm{bp}$ fragment from 15-ADON producers, respectively [21].

In the second PCR, the same DNA was amplified by the multiplex primer set developed by Ward et al. [25]. The primers for multiplex PCR were as follows: 3CON (5'-TGGCAAAGACTGGTTCAC-3'), 3D15A (5'-ACTGACCCAAGCTGCCATC-3'), 3D3A (5'-CGCATTGGCTAACACATG-3') and 3NA (5'-GTGCACAGAATATACGAGC-3') and they produce a 243 bp fragment for 3-ADON chemotypes, a 610 bp fragment for 15-ADON chemotypes and a 840 bp fragment for NIV chemotypes [25].

\subsection{PCR Conditions}

Both PCR assays were conducted using $50 \mathrm{ng}$ of fungal DNA in a total volume of $25 \mu \mathrm{L}$ containing $10 \mathrm{mM}$ Tris $\mathrm{HCl}$ ( $\mathrm{pH} \mathrm{8.0),} 1.25 \mathrm{mM} \mathrm{MgCl}_{2}, 1 \mathrm{U}$ Taq polymerase (Invitrogen, Carlsbad, CA, USA), $2.5 \mathrm{mM}$ dNTPs and $10 \mathrm{mM}$ from each primer. The PCR amplification of TRI13P1 and TRI13P2 primers consisted of an initial step at $94{ }^{\circ} \mathrm{C}$ for $4 \mathrm{~min}$, followed by 35 cycles of $94{ }^{\circ} \mathrm{C}$ for $1 \mathrm{~min}, 58{ }^{\circ} \mathrm{C}$ for $40 \mathrm{~s}, 72{ }^{\circ} \mathrm{C}$ for $40 \mathrm{~s}$, then a final extension at $72{ }^{\circ} \mathrm{C}$ for $6 \mathrm{~min}$. The PCR amplification of multiplex primers consisted of an initial step at $94{ }^{\circ} \mathrm{C}$ for $5 \mathrm{~min}$, followed by 45 cycles of $94{ }^{\circ} \mathrm{C}$ for $30 \mathrm{~s}, 52{ }^{\circ} \mathrm{C}$ for $30 \mathrm{~s}, 72{ }^{\circ} \mathrm{C}$ for $1 \mathrm{~min}$, then a final extension of $72{ }^{\circ} \mathrm{C}$ for $8 \mathrm{~min}$. Resulting PCR 
products were separated by $2 \%$ gel electrophoresis, stained with ethidium bromide (EtBr) at a final concentration of $0.2 \mu \mathrm{g} / \mathrm{mL}$ and visualized under UV light.

\subsection{Confirmation of PCR Products Using Sequencing}

A representative sample of the 120 Canadian isolates and 24 of the Chinese isolates were used for sequencing. Selected PCR products (4 Canadian 15-ADON isolates, 4 Canadian 3-ADON isolates, 5 Chinese 15-ADON isolates, 5 Chinese 3-ADON isolates and 4 Chinese NIV isolates,) were sequenced (Macrogene Corp, USA) and the sequences were subjected to multiple alignment using ClustalX (1.8) software to examine the expected deletions within the sequences.

\section{Results}

SCAR assays of all the strains from Canada and China with primer pair Fg16F/R revealed the presence of two SCAR groups, I and V. All the 3-ADON and 15-ADON chemotypes from Canada belong to the SCAR group I. All the NIV and 3-ADON chemotypes from China belong to SCAR group V. However, 15-ADON-producing isolates from China belong to either SCAR group I or $\mathrm{V}$ (Figure 1).

Figure 1. DNA of Chinese and Canadian Fusarium graminearum chemotypes amplified using Fg16 F/R primers. The 497 bp represents SCAR group V and 410 bp represents SCAR group I. Lane M: Marker; Lanes 1 and 2: NIV chemotypes, China (Fg-0921, 0905); Lanes 3 and 4: 15-ADON chemotype, China (Fg-1960, 0819); Lanes 5-7: 3-ADON chemotypes, China (Fg-0919, 0926, 0970); Lanes 8-10: 3-ADON chemotypes, Canada (M5-06-01, ON-06-39, DF-Fg-2); Lanes 11-13: 15-ADON chemotypes, Canada (DF-Fg144, ON-06-05, 55-1); Lane C: Control.

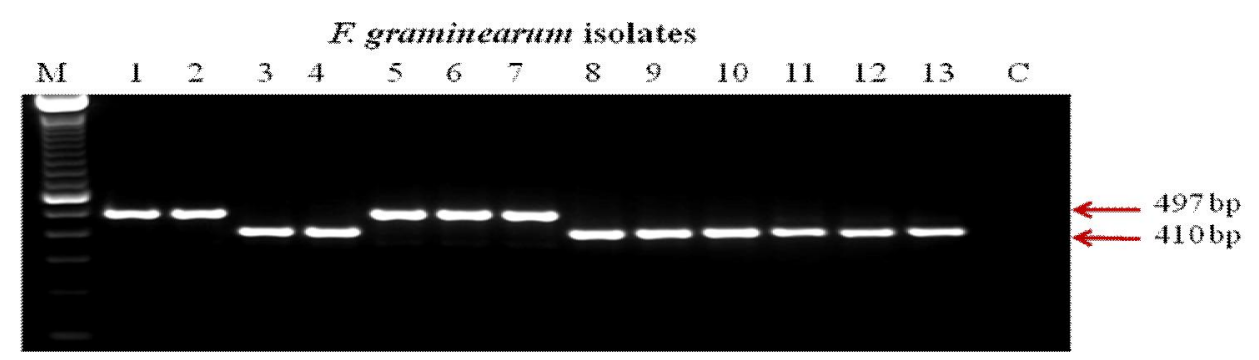

One hundred and twenty $F$. graminearum isolates from different provinces in Canada, 24 F. graminearum isolates from China were selected to examine the reliability of the TRI13P1 and TRI13P2 primers for the identification of 3-ADON and 15-ADON chemotypes in Canadian F. graminearum isolates. Our PCR assay with TRI13P1/P2 primers revealed that this single primer pair cannot be used to differentiate the two chemotypes within the Canadian isolates, although they could identify the 3-ADON, 15-ADON and NIV isolates within Chinese isolates (Figure 2A). The TRI13P1 and TRI13P2 amplified a $583 \mathrm{bp}$ fragment for all analyzed Canadian isolates regardless of whether they are $3-\mathrm{ADON}$ or $15-\mathrm{ADON}$ producers (Figure $2 \mathrm{~B}$ ). In this study all the above isolates were tested using multiplex PCR assay as well and it could differentiate the chemotypes within both Canadian and Chinese isolates. The multiplex primers amplified a $610 \mathrm{bp}$ fragment for 15-ADON 
chemotypes, a $243 \mathrm{bp}$ fragment for 3-ADON chemotypes (Figure 2C). The sequence analysis of the amplified fragments of the TRI13P1 and TRI13P2 primers of the selected 3-ADON, 15-ADON and NIV isolates from Canada, and China clearly showed the difference in TRII3 gene (Figure 3). Compared with the NIV-producers, the 3-ADON chemotypes from Canada had both the $61 \mathrm{bp}$ deletion and the $36 \mathrm{bp}$ deletion whereas the 3-ADON chemotypes from China had only the $36 \mathrm{bp}$ deletion; the 15-ADON chemotypes from both Canada and China had 36 bp and 61 bp deletions (Figure 3 ).

Figure 2. (A) DNA of Chinese Fusarium graminearum chemotype isolates amplified using TRI13P1/Tri13P2 primers [21]. The 583 bp fragments represented 15-ADON chemotypes, $644 \mathrm{bp}$ represented 3-ADON chemotypes and $858 \mathrm{bp}$ fragments represented NIV chemotypes. Lanes 1-4 and 10: 15-ADON chemotypes (Fg-0819, 1960, 0963, 0938, 0952); Lanes 8-9 and 13: 3-ADON chemotypes (Fg-0919, 0926, 0970); Lanes 5-7, 11 and 12: NIV chemotypes (Fg-0921, 0905, 0973, 0970, 0903); (B) DNA of Canadian chemotype isolates amplified using TRI13P1/Tri13P2 primers. The $583 \mathrm{bp}$ fragments represented both 3-ADON and 15-ADON chemotypes. Lanes 1, 7 and 8: 15-ADON chemotypes (PEI-0634, ON-06-05, ON-06-17); Lanes 2-6 and 9: 3-ADON chemotypes (M8-06-05, M5-06-01, SIA-06-03, PEI-06-33, ON-06-39, DF-Fg-2); (C) DNA of Canadian chemotype isolates amplified using multiplex. The $610 \mathrm{bp}$ fragments represented 15-ADON chemotypes and 243 bp fragments represented 3-ADON chemotype. Lanes 1, 7 and 8: 15-ADON chemotypes (PEI-06-34, ON-06-05, ON-06-17); Lanes 2-6 and 9: 3-ADON chemotypes (M8-06-05, M5-06-01, SIA-06-03, PEI-06-33, ON-06-39, DF-Fg-2).

A F. graminearrom isolates (China)

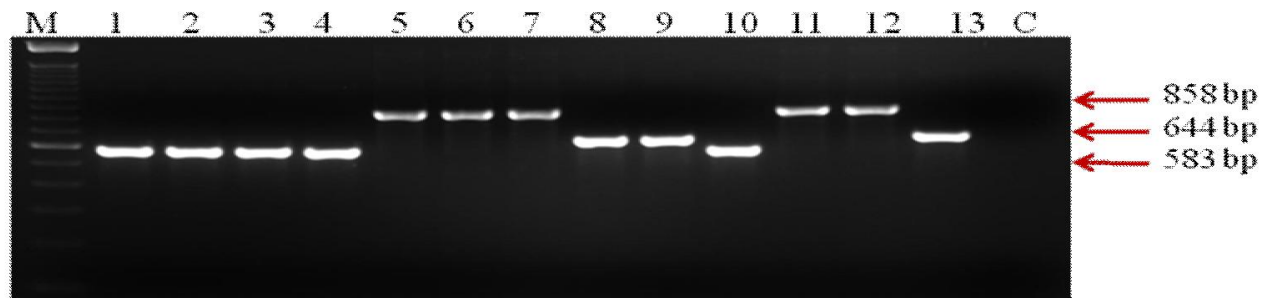

B F. graminearum isolates (Canada)

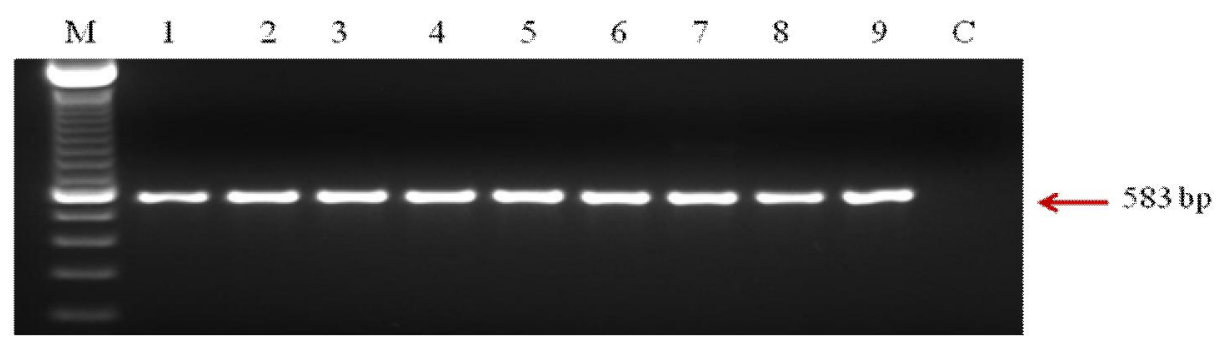

C

F. graminearnm isolates (Canada)

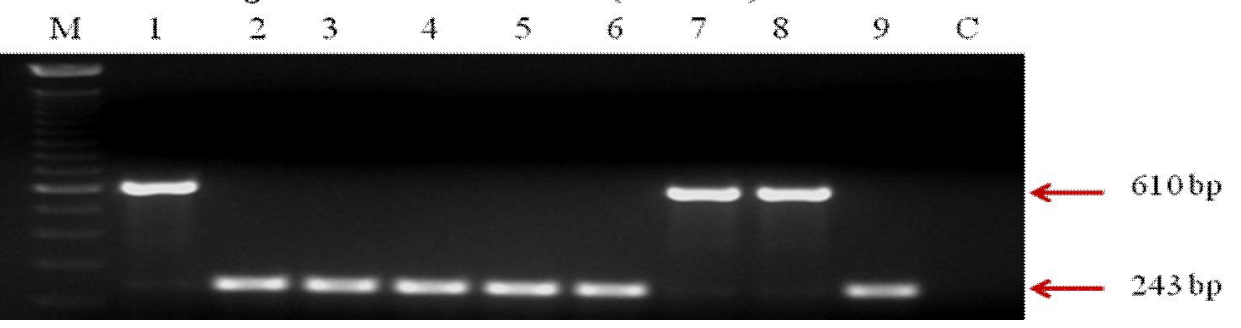


Figure 3. Alignments of TRI13 gene sequences amplified from Canadian Fusarium graminearum 3-ADON, 15-ADON chemotypes and Chinese 3-ADON, 15-ADON and NIV chemotypes. 3-ADON chemotypes, Canada (1-M8-06-05, 2-ON-06-39, 3-Q-06-32, 755-3), 15-ADON chemotypes, Canada (4-ON-06-17, 5-DF-Fg-144, 6-Q-06-10, 8-55-1), 3ADON chemotypes, China (11-Fg-0919, 12-Fg-0926, 15-Fg-0970), 15-ADON chemotypes, China (9-Fg-0819, 10-Fg-1960, 14-Fg-0963), NIV chemotypes, China (13Fg-0921, 16-Fg-0905).

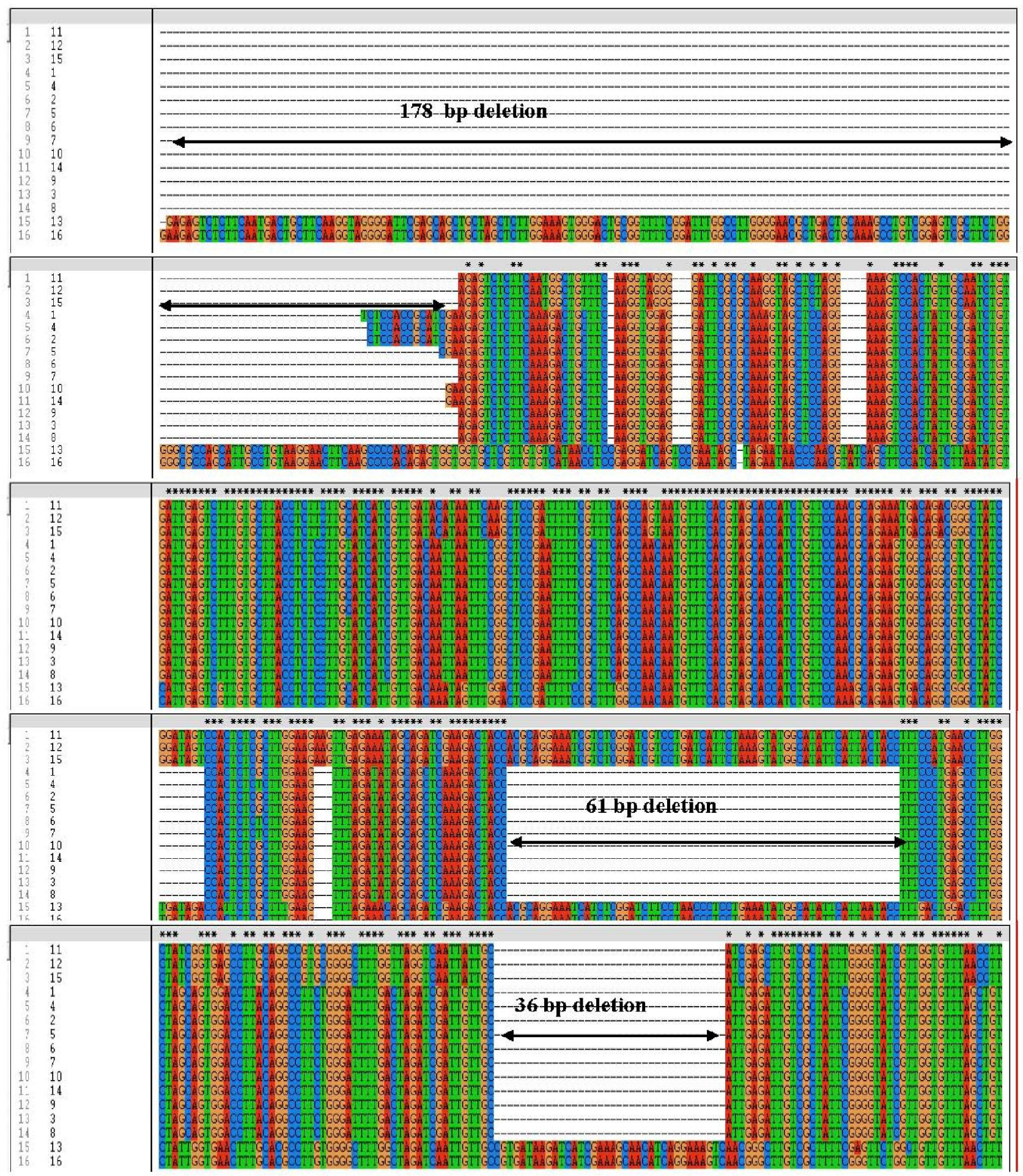




\section{Discussion}

Determination of the $F$. graminearum chemotypes using multiple primers makes the evaluation process tedious, time consuming and also they may generate false negative results $[21,26]$. Therefore use of a single primer that could differentiate the two chemotypes would accelerate the identification of chemotypes.

The TRI13 gene is functional only in NIV producers and encodes 3-acetyltrichothecene C-4 hydroxylase, enzyme that catalyses the $\mathrm{C}-4$ oxygenation of calonectrin, disruption of the gene resulted in the loss of NIV production and accumulation of DON [5,27]. The TRI13 gene consists of a unique intron of $62 \mathrm{bp}$. The TRI13P1/P2 primers were designed to identify the three chemotypes based on the deletions within the TRI13 gene (Figure 4). The chemotypic identification is based on the three deletions within the TRII3 gene found among the different chemotypes. The primers derived from TRI13 gene has been used to identify the NIV producers and DON producers. This identification was based on the largest deletion of $178 \mathrm{bp}$ fragment present only in DON producers but not in NIV producers $[5,9,28,29]$. The molecular differentiation between 3-ADON and 15-ADON chemotypes was based on the remaining two smaller deletions, $61 \mathrm{bp}$ and $36 \mathrm{bp}$ within the coding region. The $15-\mathrm{ADON}$ chemotypes have both $61 \mathrm{bp}$ and $36 \mathrm{bp}$ deletions and amplified a product of $583 \mathrm{bp}$ with TRI13P1/TRI13P2 primers; 3-ADON chemotypes have only the $36 \mathrm{bp}$ deletion and amplified a product of $644 \mathrm{bp}$. In this study, all the examined Canadian isolates (both 3-ADON and 15-ADON) had amplified a product of $583 \mathrm{bp}$, revealing that Canadian 3-ADON chemotypes have the $61 \mathrm{bp}$ deletion in the TRII3 gene which is not present in the Chinese 3-ADON chemotypes. Sequencing results clearly confirmed the $61 \mathrm{bp}$ deletion in the TRI13 gene within the Canadian 3-ADON isolates. Lee et al. [5] reported that several deletions, substitutions and insertions were found in putative TRI13 genes in DON producing strains collected from Korea, Nepal and the United states. Further, the nuclear alignment of the putative TRI13 fragments indicated that these characteristics were highly conserved among the DON-producing strains from different geographical regions.

Figure 4. (A) Diagrammatic presentations of TRI13 genes of 15-ADON, 3-ADON and NIV chemotypes in Chinese isolates of Fusarium graminearum (adopted from Wang et al. [21]); (B) Diagrammatic presentations of TRI13 genes of 15-ADON and 3-ADON chemotypes in Canadian isolates of $F$. graminearum: $\square$ - represent deletions; $\mathbb{Z}$ represent insertions in the TRI13 gene.

A

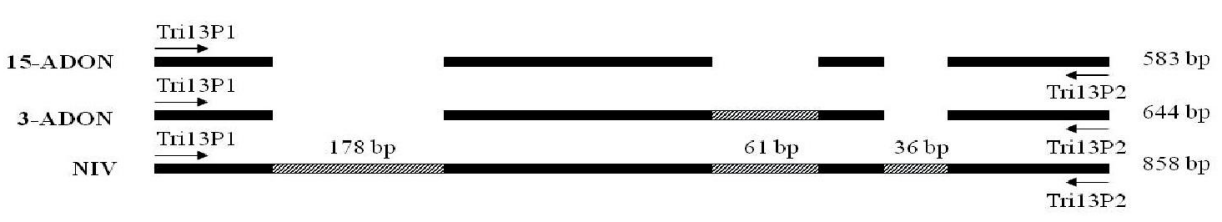

$\mathbf{B}$

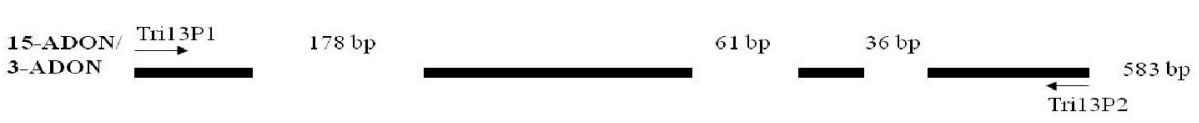


The multiplex PCR assay was based on the primers derived from trichothecene 15-O-acetyltransferase (TRI3) gene [7]. McCormick et al. [30] isolated TRI3 gene from $F$. sporotrichioides. The tri3 mutants of $F$. sporotrichiodies were able to acetylate the trichothecene $\mathrm{C}-3$ hydroxyl group but not the $\mathrm{C}-15$ hydroxyl group. These findings indicated that, acetylation of C-15 hydroxyl is mediated by TRI3 gene. Multiplex PCR assay based on TRI3 gene could differentiate the 3-ADON and 15-ADON chemotypes in the Canadian isolates that could not be differentiated using the TRI13P1/TRI13P2 primers (Figure 1A). So this confirmed that the TRI13 gene sequences of the examined Canadian 3-ADON isolates are different from that of examined Chinese 3-ADON isolates. According to the SCAR analysis the Canadian 3-ADON isolates belong to SCAR group I and Chinese 3-ADON isolates SCAR group V. The isolates from SCAR group I represent $F$. graminearum sensu stricto lineage 7 whereas group $\mathrm{V}$ represents lineage $6[16,29]$. Therefore the single primer developed by Wang et al., 2008 [21] based on the TRII3 gene cannot be used to differentiate the 3-ADON and 15-ADON chemotypes of $F$. graminearum sensu stricto lineage 7 although it could be used to differentiate the three chemotypes of $F$. graminearum in lineage 6.

It has been reported that variations in trichothecene production may be caused by the alleleic polymorphisms in the tricothecene biosynthesis gene cluster, as a result of selection pressure from environments $[7,10]$. Several authors have examined the relationship between chemotype diversity and the geographical distribution. Both DON and NIV chemotypes have been reported in Africa, Asia and Europe whereas in North America only DON chemotypes were reported [3,8,31]. But in a recent study Starkey et al. [12] identified six F. gramineaum isolates with a NIV or 3-ADON chemotype in Louisiana, USA. Also Gale et al. [32] reported that NIV type populations of $F$. graminearum and $F$. asiaticum were prevalent on wheat in Southern Louisiana. They reported a presence of high proportion of NIV type $F$. graminearum among isolates (79\%) collected from small grain growing regions of Louisiana and also NIV type isolates were identified in collections from Arkansas, North Carolina and Missouri. Studies done on phylogenetics of Fusarium spp. showed that significant levels of sexual recombination took place within the populations of the $F$. graminearum clade [10,33]. Members of all lineages are cross fertile with strains belonging to the lineage 7 and with strains in other lineages. Lineage 7 is considered as a universally cross-fertile lineage [34]. The identification of a hybrid strain between two F. graminearum clade species [10] and reports by Bowden and Leslie [33] on the laboratory out-crossing amongst the lineages provided evidences for the possibility of developing novel lineages in an appropriate geographical location. The observed genetic differences between the 3-ADON chemotype populations in Canada and China may be resulted from a random mutation (insertion/deletion) that took place in one of the populations and accumulated due to genetic drift and/or selection. Climatic conditions, crop rotations and crop management strategies also differ markedly between Canada and China; therefore these also have an influence on the spread of the populations within a particular geographical area.

The genetic diversity of the species within the $F$. graminearum clade from different geographical regions in the world may affect the current disease management, quarantine regulations and breeding strategies [11]. Therefore studies on genetic diversity of $F$. graminearum clade species are important in preventing future outbreaks of FHB epidemics. The findings from this study provide a foundation for further investigations to understand the genetic diversity of $F$. graminearum chemotypes from different geographical regions. 


\section{Acknowledgements}

We thank the financial support of NSERC Discovery Grant awarded to W.G.D.F to carry out this work. We are very grateful to Thomas Miedaner, State Plant Breeding Institute, University of Hohenheim, Germany and Todd Ward, United States Department of Agriculture, Peoria IL for providing the Canadian isolates.We also acknowledge and thank $\mathrm{Xu}$ Zhang, Hongxiang Ma and Peng Zhang, Jiangsu Academy of Agriculture Science for giving the Chinese isolates for the study. We also thank Khaled Al-Taweel for helping in manuscript preparation.

\section{References}

1. Charmley, L.L.; Trenholm, H.L.; Prelusky, D.B.; Rosenberg, A. Economic losses and decontamination. Nat. Toxins 1995, 3, 199-203.

2. Proctor, R.H.; Hohn, T.M.; McCormick, S.P. Reduced virulence of Gibberella zeae caused by disruption of a trichothecene toxin biosynthetic gene. Mol. Plant Microbe Interact. 1995, 8, 593-601.

3. Miller, J.D.; Greenhalgh, R.; Wang, Y.Z.; Lu, M. Trichothecene chemotypes of three Fusarium species. Mycologia 1991, 83, 121-130.

4. Desjardins, A.E. Trichothecenes. In Fusarium Mycotoxins-Chemistry, Genetics, and Biology; The American Phytopathological Society Press: St. Paul, MN, USA, 2006; pp. 13-64.

5. Lee, T.; Han, Y.K.; Kim, K.H.; Yun, S.H.; Lee, Y.W. Tri13 and Tri7 determine deoxynivalenoland nivalenol-producing chemotypes of Gibberella zeae. Appl. Environ. Microbiol. 2002, 68, 2148-2154.

6. Lee, T.; Oh, D.W.; Kim, H.S.; Lee, J.; Kim, Y.H.; Yun, S.H.; Lee, Y.W. Identification of deoxynivalenol- and nivalenol- producing chemotypes of Gibberella zeae by using PCR. Appl. Environ. Microbiol. 2001, 67, 2966-2972.

7. Ward, T.; Bielawski, J.P.; Kistler, C.H.; Sullivan, E.; O’Donnell, K. Ancestral polymorphism and adaptive evolution in the trichothecene mycotoxin gene cluster of phytopathogenic Fusarium. Proc. Natl. Acad. Sci.USA 2002, 99, 9278-9283.

8. Jennings, P.; Coates, M.E.; Turner, J.A.; Chandler, E.A.; Nicholson, P. Determination of deoxynivalenol and nivalenol chemotypes of Fusarium graminearum isolates from England and Wales by PCR assay. Plant Pathol. 2004, 53, 643-652.

9. Quarta, A.; Mita, G.; Haidukowski, M.; Santino, A.; Mule, G.; Visconti, A. Multiplex PCR assay for the identification of nivalenol, 3- and 15-acetyldeoxynivalenol chemotypes in Fusarium. FEMS Microbiol. Lett. 2006, 259, 7-13.

10. O’Donnell, K.; Kistler, H.C.; Tacke, B.K.; Casper, H.H. Gene genealogies reveal global phylogeographic structure and reproductive isolation among lineages of Fusarium graminearum, the fungus causing wheat scab. Proc. Natl. Acad. Sci. USA 2000, 97, 7905-7910.

11. O’Donnell, K.; Ward, T.J.; Geiser, D.M.; Kistler, H.C.; Aoki, T. Genealogical concordance between the mating type locus and seven other nuclear genes supports formal recognition of nine phylogenetically distinct species within the Fusarium graminearum clade. Fungal Genet. Biol. 2004, 41, 600-623. 
12. Starkey, D.E.; Ward, T.J.; Aoki, T.; Gale, L.R.; Kistler, H.C.; Geiser, D.M.; Suga, H.; Tóth, B.; Varga, J.; O’Donnell, K. Global molecular surveillance reveals novel Fusarium head blight species and trichothecene toxin diversity. Fungal Genet. Biol. 2007, 44, 1191-1204.

13. O’Donnell, K.; Ward, T.J.; Aberra, D.; Kistler, H.C.; Aoki, T.; Orwig, N.; Kimura, M.; Bjørnstad, A.; Klemsdal, S.S. Multilocus genotyping and molecular phylogenetics resolve a novel head blight pathogen within the Fusarium graminearum species complex from Ethiopia. Fungal Genet. Biol. 2008, 45, 1514-1522.

14. Yli-Mattila, T.; Gagkaeva, T.; Ward, T.J.; Aoki, T.; Kistler, H.C.; O’Donnell, K. A novel Asian clade within the Fusarium graminearum species complex includes a newly discovered cereal head blight pathogen from the Russian Far East. Mycologia 2009, 101, 841-852.

15. Goswami, R.S.; Kistler, H.C. Pathogenicity and in planta mycotoxin accumulation among members of the Fusarium graminearum species complex on wheat and rice. Phytopathology 2005, 95, 1397-1404.

16. Zhang, J.B.; Li, H.P.; Dang, F.J.; Qu, B.; Xu, Y.B.; Zhao, C.S.; Liao, Y.C. Determination of the trichothecene mycotoxin chemotypes and associated geographical distribution and phylogenetic species of the Fusarium graminearum clade from China. Mycol. Res. 2007, 111, 967-975.

17. Cumagun, C.J.R.; Bowden, R.L.; Jurgenson, J.E.; Leslie, J.F.; Miedaner, T. Genetic mapping of pathogenicity and aggressiveness of Gibberella zeae (Fusarium graminearum) toward wheat. Phytopathology 2004, 94, 520-526.

18. Qu, B.; Li, H.P.; Zhang, J.B.; Xu, Y.B.; Huang, T.; Wu, A.B.; Zhao, C.S.; Carter, J.; Nicholson, P.; Liao, Y.C. Geographic distribution and genetic diversity of Fusarium graminearum and F. asiaticum on wheat spikes throughout China. Plant Pathol. 2008, 57, 15-24.

19. Bai, G.H.; Shaner, G. Variation in Fusarium graminearum and cultivar resistance to wheat scab. Plant Dis. 1996, 80, 975-979.

20. Brasier, C.M.; Cooke, D.E.L.; Duncan, J.M. Origin of a new Phytophthora pathogen through interspecific hybridization. Proc. Natl. Acad. Sci. USA 1999, 96, 5878-5883.

21. Wang, J.; Li, H.; Qu, B.; Zhang, J.; Huang, T.; Chen, F.; Liao, Y. Development of a generic PCR detection of 3-acetyldeoxynivalenol, 15-acetyldeoxynivalenol- and nivalenol-chemotypes of Fusarium graminearum Clade. Int. J. Mol. Sci. 2008, 9, 2495-2504.

22. Guo, X.W.; Fernando, W.G.D.; Seow-Brock, H.Y. Population structure, chemotype diversity, and potential chemotype shifting of Fusarium graminearum in wheat fields of Manitoba. Plant Dis. 2008, 92, 756-762.

23. Fernando, W.G.D.; Zhang, J.K.; Dusabenyagasani, M.; Guo, X.; Ahmed, H.; McCallum, B. Genetic diversity of Gibberella zea isolates from Manitoba. Plant Dis. 2006, 90, 1337-1342.

24. Carter, J.P.; Razanoor, H.N.; Desjardins, A.E.; Nicholson, P. Variation in Fusarium graminearum isolates from Nepal associated with their host of origin. Plant Pathol. 2000, 49, 1-10.

25. Ward, T.J.; Clear R.M.; Rooney, A.P.; O’Donnell, K.; Gaba, D.; Patrick, S.; Starkey, D.; Gilbert, J.; Geiser, D.; Nowicki, T. An adaptive evolutionary shift in Fusarium head blight pathogen populations is driving the rapid spread of more toxigenic Fusarium graminearum in North America. Fungal Genet. Biol. 2008, 25, 473-484. 
26. Li, H.P.; Wu, A.B.; Zhao, C.S.; Scholten, O.; Löffler, H.; Liao, Y.C. Development of a generic PCR detection of deoxynivalenol- and nivalenol-chemotypes of Fusarium graminearum. FEMS Microbiol. Lett. 2005, 243, 505-511.

27. Brown, D.W.; McCormick, S.P.; Alexander, N.J.; Proctor, R.H.; Desjardins, A.E. Inactivation of a cytochrome P-450 is a determinant of trichothecene diversity in Fusarium species. Fungal Genet. Biol. 2002, 36, 224-233.

28. Brown, D.W.; McCormick, S.P.; Alexander, N.J.; Proctor, R.H.; Desjardins, A.E. A genetic and biochemical approach to study trichothecene diversity in Fusarium sporotrichioides and Fusarium graminearum. Fungal Genet. Biol. 2001, 32, 121-133.

29. Chandler, E.A.; Simpson, D.R.; Thomsett, M.A.; Nicholson, P. Development of PCR assays to Tri7 and Tri 13 trichothecene biosynthetic genes, and characterisation of chemotypes of Fusarium graminearum, Fusarium culmorum and Fusarium cerealis. Physiol. Mol. Plant Pathol. 2003, 62, 355-367.

30. McCormick, S.P.; Hohn, T.M.; Desjardins, A.E. Isolation and characterization of Tri3, a gene encoding 15-O-acetyltransferase from Fusarium sporotrichioides. Appl. Environ. Microbiol. 1996, 62, 353-359.

31. Mirocha, C.J.; Abbas, H.K.; Windels, C.E.; Xie, W. Variation in deoxynivalenol, 15-acetyldeoxynivalenol, 3-acetyldeoxynivalenol, and zearalenone production by Fusarium graminearum isolates. Appl. Environ. Microbiol. 1989, 55, 1315-1316.

32. Gale, L.R.; Harrison, S.A.; Ward, T.J.; O’Donnell, K.; Milus, E.A.; Gale, S.W.; Kistler, H.C. Nivalenol-type populations of Fusarium graminearum and F. asiaticum are prevalent on wheat in southern Louisiana. Phytopathology 2010, 101, 124-134.

33. Bowden, R.L.; Leslie, J.F. Sexual recombination in Gibberella zeae. Phytopathology 1999, 89, 182-188.

34. Bowden, R.L.; Leslie, J.F.; Lee, J.; Lee, Y.W. Cross Fertility of Lineages in Fusarium graminearum (Gibberella zeae). In The Global Fusarium Initiative for International Collaboration: A Strategic Planning Workshop; Ban, T., Lewis, J.M., Phipps, E.E., Eds.; CIMMYT: El Batan, Mexico, 2006; pp. 54-60.

(C) 2011 by the authors; licensee MDPI, Basel, Switzerland. This article is an open access article distributed under the terms and conditions of the Creative Commons Attribution license (http://creativecommons.org/licenses/by/3.0/). 